
$\angle$ Research Square
Preprints are preliminary reports that have not undergone peer review.
They should not be considered conclusive, used to inform clinical practice, or referenced by the media as validated information.

\title{
In Vitro Anticancer Activity of Five Marine Seaweeds Extract From Egypt Against Human Breast and Colon Cancer Cell Lines
}

\author{
Jelan Mofeed \\ Suez University \\ Mohamed Deyab \\ Damietta University Faculty of Science \\ Abd El-Naser Sabry \\ Damietta University Faculty of Science \\ Fatma Ward ( Fatma2028@yahoo.com ) \\ Damietta University https://orcid.org/0000-0003-2238-9013
}

\section{Research Article}

Keywords: Anticancer, Cytotoxic Activity, HCT-116, IC50, MCF-7, Seaweeds

Posted Date: May 6th, 2021

DOI: https://doi.org/10.21203/rs.3.rs-462221/v1

License: () (1) This work is licensed under a Creative Commons Attribution 4.0 International License. Read Full License 


\section{Abstract}

Ulva fasciata, U. lactuca, Amphiroa anceps, Corallina mediterranea, and Sargassum filipendula extract were screened for their in vitro anticancer activity against human breast adenocarcinoma cell line (MCF-7) and colorectal carcinoma cell line (HCT-116). Algal extracts exhibited significantly dose-dependent anticancer activity without significant cytotoxicity against normal human fibroblasts. The maximum inhibitory percentages against MCF-7 and HCT-116 cell lines were recorded by $U$. lactuca $(88.5 \pm 1.08 \%)$ and $A$. anceps $(86.1 \pm 2.88 \%)$ extracts. Algal extracts showed cytotoxic effect against MCF-7 cell line with fifty percent inhibitory concentration $\left(\mathrm{IC}_{50}\right)$ values ranging from $3.54 \pm 1.2$ to $21.2 \pm 1.1 \mu \mathrm{g} \mathrm{mL}^{-1}$. The seaweeds extract has a less cytotoxic effect against $\mathrm{HCT}$ 116. FTIR and GC-MS analyses of the extracts indicated that the anticancer potential of the tested seaweeds may be returned to the presence of various anticancer compounds such as palmitic acid, oleic acid, retinoic acid, dihydroactinidiolide, thiosemicarbazide, diisobutyl phthalate, and phytol. Thus, seaweed extracts may be a promising natural source of safe anticancer agents against human breast and colon cancers.

\section{Introduction}

Macroalgae were classified into three broad groups as green algae (Chlorophyta), brown algae (Phaeophyta), and red algae (Rhodophyta). For many ages, algae have been considered a basket for many applicable compounds in many fields including pharmaceutical products (Mofeed and Mosleh, 2013). Seaweeds known as marine macroalgae are gaining significant importance due to their production of novel medically active substances like fatty acids, alkaloids, polysaccharides, cyclic peptides, heterocyclic carbons, terpenes, and stereos. Most of these substances display a broad range of biological activities such as anticancer, anti-inflammatory, antimicrobial, antioxidant, antiviral, and antimalarial (Mofeed et al., 2019; Deyab et al., 2020).

Cancer is considered one of the most diseases threatening the survival of humanity nowadays and there is no main reason for it. In all cancer types, some of the body's cells begin to divide without stopping, forming an uncontrolled cell growth that spread into surrounding tissues and can concurrently start anywhere in the human body (Yousefi et al., 2018; Mofeed et al., 2018).

Breast cancer is the first most common malignant tumor in women and the second in the world (Ilhan et al., 2017). It is the most likely cause of cancer deaths in women worldwide. Incident of breast carcinoma has been increased from 1.7 million in 2005 to 2.4 million cases in 2015 (Fitzmaurice et al., 2017 ). In Egypt, breast cancer occupied the top rank and constitutes $32 \%$ among women (Ibrahim et al., 2014).

Colon cancer known as colorectal cancer is the third most common cancer in the world with an estimated 1.4 million cases diagnosed and 693,900 deaths in 2012 occurring worldwide. It is the second-leading cause of cancer deaths in both men and women (Torre et al., 2015). In Egypt, colorectal cancer is the 7th commonest cancer, representing $3.47 \%$ of male cancers and $3 \%$ of female cancers (Ibrahim et al., 2014).

Till now, cancer treatments do not have safe medicine as the currently available drugs are causing side effects like vomiting, diarrhea, fatigue, and nausea. Hence, it is important to explore and identify new safe, cheap, and less toxic anticancer agents from natural sources. Most pharmaceutical products have been derived from various microorganisms, herbal plants, and seaweeds (Mofeed, 2019).

Macroalgae can be seen as natural factories for producing bioactive compounds. A sulfated polysaccharide (fucoidan) extracted from the brown seaweeds, Fucus spp., Sargassum spp., Turbinaria spp. and Padina spp. has been shown anti-carcinogenic activity against both colon and breast cancer cell lines (Isnansetyo et al., 2017). Numerous studies have reported anticancer activity of various compounds extracted from Laminaria digitata, Cymopolia barbata, Lithothamnion calcareum, Undaria pinnatifida against HT-29 colon cancer cells (Yang et al., 2013; Badal et al., 2012). Moreover, some compounds isolated from chlorophytes have been exhibited cytotoxic activity against breast, skin, lung, and prostate cancer lines (Tavares-Carreón et al., 2020).

Some marine macroalgal species, collected from Egypt such as Ulva fasciata and U. lactuca (Chlorophyta), Amphiroa anceps, and Corallina mediterranea (Rhodophyta), and Sargassum filipendula (Phaeophyta) have been sufficiently unexplored as anticancer drugs. Therefore, the main objective of the present study is to analyze the composition of these five seaweeds using FTIR and GC-MS analyses to screen their metabolites for in vitro anticancer potentialities against breast cancer adenocarcinoma cell line (MCF-7) and colon carcinoma cell line (HCT-116).

\section{Materials And Methods}

\subsection{Collection of seaweed samples}

Five seaweeds were collected from Egypt's shores by hand-picking during low tide in October 2016. Ulva fasciata and Amphiroa anceps were collected from Alexandria seashore - the Mediterranean Sea, Egypt (Abu-Qir 31 $19 \mathrm{~N}, 30^{\circ} 03 \mathrm{E}$ ), Ulva lactuca and Corallina mediterranea were collected from Damietta

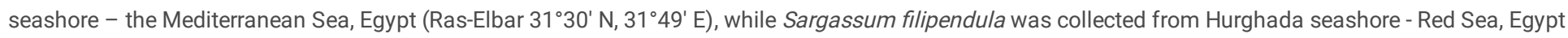
(Hurghada $27^{\circ} 13^{\prime} \mathrm{N}, 33^{\circ} 45^{\prime} \mathrm{E}$ ) (Fig. 1). Seaweed species were taxonomically identified according to Braune and Guiry (2011) and Jha et al. (2009). Photos of the collected seaweeds were shown in Fig. 2.

\subsection{Preparation of seaweed samples}

After collection, fronds of seaweeds were washed several times with tap water and then with distilled water to remove epiphytes, sand, and salts. Seaweeds were spread on paper to remove excess water and shade-dried at room temperature. Finally, $100 \mathrm{~g}$ of dry samples were ground into 2 mm and stored in airtight brown glass containers at room temperature for further analysis.

\subsection{Extraction methods}


The dried seaweed sample $(25 \mathrm{~g})$ was extracted using $250 \mathrm{ml}$ methanol: hexane (1:1) at $65^{\circ} \mathrm{C}$ in the Soxhlet apparatus. The extraction procedure was repeated until the extract was clear (most compounds were completely extracted). The extraction process of U. fasciata, U. lactuca, A. anceps, C. mediterranea, and $S$. filipendula has run for $22,24,16,18$, and 16 hours, respectively. The liquid extracts were then cooled and concentrated using a rotary evaporator at $30-45^{\circ} \mathrm{C}$. Extracts were stored in labeled sterile screw-capped bottles at $4^{\circ} \mathrm{C}$ until it was tested. Methanol and hexane were of analytical grade and were purchased from Sigma Aldrich, USA.

\subsection{In vitro anticancer activity}

\subsubsection{Cell lines}

Human breast adenocarcinoma cell line (MCF-7), Human colon carcinoma cell line (HCT-116), and normal human fibroblast cells were obtained from the American Type Culture Collection (ATCC, Minnesota, USA) and were maintained at the National Cancer Institute, Cairo, Egypt, by serial sub-culturing. MCF-7 cells were cultured in Dulbecco's Modification of Eagle's Medium (DMEM, Sigma Aldrich, USA) while, HCT-116 cells were cultured in McCoy's 5a medium (Sigma-Aldrich, USA). Both media were supplemented with antibiotic-free 10\% Fetal Bovine Serum (FBS, Sigma., USA), 100 U mL ${ }^{-1}$ penicillin, and 2 mg mL ${ }^{-1}$ streptomycin. The cells were maintained at $37^{\circ} \mathrm{C}$ in $95 \%$ relative humidified atmosphere containing $5 \% \mathrm{CO}_{2}$.

\subsubsection{Anticancer activity of organic extract of seaweeds using Sulforhodamine B (SRB) assay}

After seaweed extraction, we get rid of methanol: hexane solvent and dissolve it in DMSO for further analyses. Various concentrations $(0,12.5,25,50$, and 100 $\mu \mathrm{g} \mathrm{mL}^{-1}$ ) of organic seaweeds extract were prepared for the following in vitro anticancer assay. $0.5 \%$ (v/v) DMSO was used as a control sample.

In vitro anticancer assay of the algal extracts against MCF-7, HCT-116, and fibroblast normal cells were performed using a SRB assay as it is a sensitive method for evaluating cytotoxic activity (Skehan et al., 1990). All determinations were replicated three times in a day and the mean values were recorded. IC ${ }_{50}$ (the extract concentration that resulted in $50 \%$ of cell growth inhibition) was calculated using the Prism program (Graph Pad prim version 7.0 software at a $95 \%$ confidence limit.). Also, the percent of cell inhibition (death) was calculated using the following formula:

Percent cell inhibition $(\%)=100-($ Absorbance of sample/Absorbance of control $\times 100)$

\subsection{Identification of phytochemicals in the algal extracts}

\subsubsection{Fourier transforms infrared spectrometry (FTIR) analysis}

The FTIR characterization of powdered seaweeds was carried out with a Mattson 5000 FTIR spectrometer. Two mg of seaweed powder was ground with approximately $100 \mathrm{mg}$ of $\mathrm{KBr}$ in a mortar until the mixture was formed in fine particles and then pressed as a disc (10 mm) for transforming IR spectral measurements in the frequency range of $4000-400 \mathrm{~cm}^{-1}$ at $25^{\circ} \mathrm{C}$.

\subsubsection{GC-MS analysis}

Gas Chromatography-Mass Spectrometry (GC-MS) analysis of methanol: hexane extracts of the tested seaweeds were performed using Varian Chrompack CP-3800 GC/MS/MS-2000 equipped with split-splitless injector and DB-5.625 GC column ( $30 \mathrm{~m} \times 0.25 \mathrm{~mm}$ i.d., $0.25 \mu \mathrm{m}$ film thickness). An electron ionization system with ionization energy of $70 \mathrm{eV}$ was used. The injector temperature was set at $275^{\circ} \mathrm{C}$ for 5 min with injection of $5 \mu$ l volumes of extracts. A linear temperature program was adapted to separate the different components as follows: initially, the column maintained at $40^{\circ} \mathrm{C}$ for $3.5 \mathrm{~min}$, ramped at a rate of $10^{\circ} \mathrm{C} \mathrm{min}-1$ to $250^{\circ} \mathrm{C}$ at which it was held isothermal for $10 \mathrm{~min}$; a second ramp $\left(20^{\circ} \mathrm{C} \mathrm{min}{ }^{-1}\right)$ was then applied to $280^{\circ} \mathrm{C}$ and held isothermal for $5 \mathrm{~min}$, the total run time was $75.5 \mathrm{~min}$. The temperatures of the transfer line and ion source were maintained at $250^{\circ} \mathrm{C}$ and $200^{\circ} \mathrm{C}$, respectively. Identification of the compounds was performed based on the comparison of their relative retention time (Rt) and mass spectra with those of the National Institute of Standards and Technology (NIST) and Wiley MS spectra libraries data of the GC/MS system. Also, the percent relative peak area of the identified components was estimated.

\subsection{Statistical analysis}

Data were analyzed by two-way analysis of variance (ANOVA) using SPSS version 22 . The difference was considered significant when $p<0.05$. All determinations were carried out in triplicates and the results are expressed as a mean \pm standard deviation (SD).

\section{Results}

\subsection{Cytotoxicity activity of seaweeds extracts}

Different concentrations $\left(12.5,25,50\right.$, and $100 \mu \mathrm{g} \mathrm{mL}^{-1}$ ) of organic extract (methanol: hexane) of U. fasciata, U. lactuca, A. anceps, C. mediterranea, and $S$. filipendula were applied to SRB assay to test their anticancer potential. Also, IC $\mathrm{I}_{50}$ was calculated for all extracts against both MCF-7 and HCT-116 cell lines. To evaluate the cytotoxic effect of the tested extracts against both MCF-7 and HCT-116 cell lines, the activity of these extracts was also examined using human fibroblast cells. All the tested extracts did not affect the viability of normal fibroblast cells.

As shown in Table 1, two-way ANOVA showed that there were significant effects of seaweeds extracts and their concentrations on both MCF-7 and HCT-116 cell lines. The results indicated that seaweeds extracts at different concentrations exhibited significant dose-dependent inhibitory effects $(p<0.005)$ against Loading [MathJax]/jax/output/CommonHTML/fonts/TeX/fontdata.js sure (Table 2 and Fig. 3-4). The highest percentage of growth inhibition against MCF-7 was $88.5 \pm$ 
$1.08 \%$ by $100 \mu \mathrm{g} \mathrm{mL}^{-1}$ of $U$. lactuca extract, followed by $U$. fasciata $(86.1 \pm 1.73 \%)$ and $A$. anceps $(85.8 \pm 1.14 \%)$. On HCT- 116 cancer cell line, the highest inhibition was represented by $A$. anceps, followed by $C$. mediterranea.

Table 1

Two-way ANOVA shows the effect of the main factors (extract of seaweeds and concentration of extracts) and their interaction on inhibition percentage of MCF-7 and HCT-116 cell

\begin{tabular}{|lccc|}
\hline \multicolumn{4}{|c|}{ lines. } \\
\hline Source of variation & df & F & P \\
\hline Seaweed extract & MCF-7 cell line \\
\hline Concentrations of extract & 3 & 34.4 & Significant \\
\hline Seaweed extract & HCT-116 cell line \\
\hline Concentrations of extract & 3 & 30.6 & Significant \\
\hline Where df: degrees of freedom, F: F-statistics, P: significance. \\
\hline
\end{tabular}

Table 2

Cell inhibition (\%) and $\mathrm{IC}_{50}$ values of extracts of five seaweeds against MCF-7 and HCT-116 cell lines. Each value is the mean of three replicates \pm S[

\begin{tabular}{|c|c|c|c|c|c|c|c|c|c|c|}
\hline \multirow{3}{*}{$\begin{array}{l}\text { Concentration } \\
\left(\mu \mathrm{g} \mathrm{mL}^{-1}\right)\end{array}$} & \multicolumn{5}{|c|}{ Cell inhibition (\%) against MCF-7 cell line line after 48 hours } & \multicolumn{5}{|c|}{ Cell inhibition (\%) against HCT-116 cell line after 48 hours } \\
\hline & & & A. & C. mediterranea & & & & A. & C. mediterranea & $S$. \\
\hline & fasciata & lactuca & anceps & & Filipendula & fasciata & lactuca & anceps & & filip \\
\hline 0 & 0 & 0 & 0 & 0 & 0 & 0 & 0 & 0 & 0 & \\
\hline 12.5 & $\begin{array}{l}63.6 \pm \\
0.86\end{array}$ & $76.7 \pm 3.11$ & $61.9 \pm 1.68$ & $53.6 \pm 1.45$ & $\begin{array}{l}43.6 \\
\pm 0.89\end{array}$ & $\begin{array}{l}18.9 \pm \\
1.81\end{array}$ & $24.6 \pm 1.07$ & $42.3 \pm 2.25$ & $16.1 \pm 0.92$ & \\
\hline 25 & $\begin{array}{l}76.2 \pm \\
1.31\end{array}$ & $84.3 \pm 1.62$ & $72.2 \pm 0.71$ & $61.3 \pm 2.41$ & $\begin{array}{l}51.3 \\
\pm 1.25\end{array}$ & $\begin{array}{l}33.8 \pm \\
2.40\end{array}$ & $55.4 \pm 0.85$ & $78.6 \pm 1.65$ & $73.1 \pm 1.37$ & \\
\hline 50 & $\begin{array}{l}79.8 \pm \\
1.09\end{array}$ & $84.8 \pm 0.79$ & $83.9 \pm 2.05$ & $64.6 \pm 1.93$ & $\begin{array}{l}60.2 \\
\pm 1.66\end{array}$ & $\begin{array}{l}56.9 \pm \\
1.21\end{array}$ & $65.4 \pm 1.62$ & $85.4 \pm 3.12$ & $78.9 \pm 2.23$ & \\
\hline 100 & $\begin{array}{l}86.1 \pm \\
1.73\end{array}$ & $88.5 \pm 1.08$ & $85.8 \pm 1.14$ & $66.4 \pm 2.19$ & $\begin{array}{l}65.4 \\
\pm 2.48\end{array}$ & $\begin{array}{l}73.1 \pm \\
2.75\end{array}$ & $78.7 \pm 1.03$ & $86.1 \pm 2.88$ & $79.1 \pm 1.47$ & \\
\hline $\begin{array}{l}\mathrm{IC}_{50}(\mu \mathrm{g} \mathrm{mL}- \\
\left.{ }^{-}\right)\end{array}$ & $\begin{array}{l}6.53 \pm \\
1.3\end{array}$ & $3.54 \pm 1.2$ & $7.42 \pm 0.8$ & $9.98 \pm 0.5$ & $\begin{array}{l}21.2 \\
\pm 1.1\end{array}$ & $\begin{array}{l}40.9 \pm \\
1.5\end{array}$ & $23.4 \pm 1.1$ & $13.9 \pm 1.2$ & $17.6 \pm 1.3$ & \\
\hline
\end{tabular}

All seaweeds extracts inhibited MCF-7 cells significantly with IC ${ }_{50}$ ranging from $3.54 \pm 1.2 \mu \mathrm{g} \mathrm{mL}^{-1}$ by $U$. lactuca to $21.2 \pm 1.1 \mu \mathrm{g} \mathrm{mL}-1$ by $S$. filipendula. On the other hand, all extracts showed less cytotoxicity on HCT-116 cell lines with $\mathrm{IC}_{50}$ ranging from $13.9 \pm 1.2 \mu \mathrm{g} \mathrm{mL}-1$ by $A$. anceps to $40.9 \pm 1.5 \mu \mathrm{g} \mathrm{mL}-1$ by $U$. fasciata. Higher cytotoxicity against MCF-7 cells was shown by extracts from Chlorophytes, U. lactuca and U. fasciata with IC ${ }_{50}$ of $3.54 \pm 1.2$ and $6.53 \pm 1.3 \mu \mathrm{g}$ $\mathrm{mL}^{-1}$, respectively. While Rhodophyta species $A$. anceps exhibited higher cytotoxicity against HCT-116 cells with $\mathrm{IC}_{50}$ of $13.9 \pm 1.2 \mu \mathrm{g} \mathrm{mL}-1$. Organic extract of Phaeophyta, S. filipendula exhibited less cytotoxicity on both MCF-7 and HCT-116 cell lines (Table 2 and Fig. 3-4).

\subsection{Identification of phytochemicals in the algal extracts}

\subsubsection{FT-IR characterization}

FTIR analyses of $U$. fasciata, $U$. lactuca, $A$. anceps, $C$. mediterranea, and $S$. filipendula were performed to determine the presence of natural compounds in their crude powder. The characteristic bands are listed in Table 3. These results showed differences between the compositions of crude powdered seaweeds. 
Table 3

FT-IR absorption frequencies $\left(\mathrm{cm}^{-1}\right)$, intensity estimation, and functional group of some seaweeds collected from Egypt.

\begin{tabular}{|c|c|c|c|c|c|c|c|c|c|c|c|}
\hline \multicolumn{10}{|c|}{ Seaweeds } & \multirow[t]{3}{*}{ Functional Groups } & \multirow[t]{3}{*}{ Compound } \\
\hline \multicolumn{2}{|c|}{ U. fasciata } & \multicolumn{2}{|l|}{ U. lactuca } & \multicolumn{2}{|l|}{ A. anceps } & \multicolumn{2}{|c|}{ C. mediterranea } & \multicolumn{2}{|c|}{ S. filipendula } & & \\
\hline $\begin{array}{l}\text { A.F. }\left(\mathrm{cm}^{-}\right. \\
\text {1) }\end{array}$ & I.E. & $\begin{array}{l}\text { A.F. }\left(\mathrm{cm}^{-}\right. \\
\text {1) }\end{array}$ & I.E. & $\begin{array}{l}\text { A.F. }\left(\mathrm{cm}^{-}\right. \\
\text {1) }\end{array}$ & I.E. & $\begin{array}{l}\text { A.F. }\left(\mathrm{cm}^{-}\right. \\
\text {1) }\end{array}$ & I.E. & $\begin{array}{l}\text { A.F. }\left(\mathrm{cm}^{-}\right. \\
\text {1) }\end{array}$ & I.E. & & \\
\hline 3349 & S & 3447 & S & 3438 & $S$ & 3444 & S & 3448 & S & • O-H Stretching & •Alcohol \\
\hline 2923 & W & 2900 & W & 2923 & W & 2900 & W & 2923 & W & $\begin{array}{l}\text { - } \mathrm{CH}_{2} \text { antisymmetric stretch of methyl } \\
\text { groups }\end{array}$ & • lipids \\
\hline 2500 & W & - & - & 2524 & M & 2523 & W & - & - & - S-H thiols stretching & $\begin{array}{l}\text { - Sulfur } \\
\text { compound }\end{array}$ \\
\hline 2113 & W & 2050 & W & 2083 & W & 2100 & W & - & & $\cdot \mathrm{C} \equiv \mathrm{C}$ stretching & •Alkyne \\
\hline 1642 & S & 1642 & S & 1799 & W & 1800 & W & 1638 & W & $\begin{array}{l}\cdot \mathrm{C}=\mathrm{C} \text { stretching } \\
\cdot \mathrm{C}=\mathrm{O} \text { stretching }\end{array}$ & $\begin{array}{l}\text { - Phenyl } \\
\text { compound } \\
\text { - Amide }\end{array}$ \\
\hline- & - & - & - & 1508 & W & - & - & - & - & $\cdot \mathrm{C}=\mathrm{C}$ ( in ring) Stretching & • Lignin \\
\hline 1454 & W & 1425 & W & - & - & 1411 & S & 1421 & W & $\begin{array}{l}\cdot \mathrm{S}=\mathrm{O} \text { stretching } \\
\cdot \mathrm{O}-\mathrm{H} \text { bending }\end{array}$ & $\begin{array}{l}\text { - Sulfate } \\
\text { - Carboxylic acid }\end{array}$ \\
\hline 1108 & W & 1099 & M & - & - & - & - & 1081 & w & $\begin{array}{l}\cdot \mathrm{C}-\mathrm{O} \text { stretching } \\
\left(\mathrm{ACH}_{2} \mathrm{OH} \text { groups }\right)\end{array}$ & - Carbohydrate \\
\hline- & - & - & - & 1028 & M & 1034 & M & - & - & - C-N stretching & -Amine \\
\hline 872 & W & - & - & 876 & $S$ & 876 & $S$ & 877 & W & - $\mathrm{C}-\mathrm{H}$ bending & $\begin{array}{l}\cdot 1,2,4- \\
\text { trisubstituted }\end{array}$ \\
\hline 669 & W & 669 & W & 718 & M & 718 & M & 714 & W & - $\mathrm{C}=\mathrm{S}$ Stretching & - Sulfide \\
\hline
\end{tabular}

\subsubsection{GC-MS analysis}

The chemical constituents of the crude extracts of the tested seaweeds were analyzed using GC-MS, and the identified compounds with their molecular formula, peak area, and the chemical group were presented in Tables $4-8$. A total of 20,12, 12, 19, and 23 different compounds were identified in $U$. fasciata, $U$. lactuca, $A$. anceps, $C$. mediterranea, and $S$. filipendula, respectively. The identified compounds in the tested seaweed extracts belong to eleven main chemical groups (esters, hydrocarbons, terpenes, ketones, fatty acids, alcohols, steroids, aldehyde, amides, amines, and phenols). 8-Heptadecene, Diisobutyl phthalate, and Octadecanal, 2-bromo- were the major compounds in extracts of $U$. fasciata, $U$. lactuca, and $S$. filipendula, respectively. Whereas 1,2Benzenedicarboxylic acid, diisooctyl ester was the major compound in extracts of both $A$. anceps, and C. mediterranea. 
Phytocomponents identified in the organic extract $U$. fasciata by GC-MS

\begin{tabular}{|c|c|c|c|c|}
\hline NO. & Compound & Molecular Formula & Peak area \% & Chemical group \\
\hline 1 & Hydroxylamine, O-decyl- & $\mathrm{C}_{10} \mathrm{H}_{23} \mathrm{NO}$ & 1.3 & Amine \\
\hline 2 & 1,2-Bis(trimethylsilyl) benzene & $\mathrm{C}_{12} \mathrm{H}_{22} \mathrm{Si}_{2}$ & 1 & Hydrocarbon \\
\hline 3 & Trimethylsilyl 3-methoxy-2-(2-oxo-2-((trimethylsilyl)oxy) ethoxy) benzoate & $\mathrm{C}_{16} \mathrm{H}_{26} \mathrm{O}_{6} \mathrm{Si}_{2}$ & 0.46 & Hydrocarbon \\
\hline 4 & Benzoic acid, methyl ester & $\mathrm{C}_{8} \mathrm{H}_{8} \mathrm{O}_{2}$ & 0.71 & Ester \\
\hline 5 & Octadecane, 6-methyl- & $\mathrm{C}_{19} \mathrm{H}_{40}$ & 0.92 & Hydrocarbon \\
\hline 6 & N-Hydroxy-4-pyridinecarboxamide & $\mathrm{C}_{6} \mathrm{H}_{6} \mathrm{~N}_{2} \mathrm{O}_{2}$ & 2.1 & Amide \\
\hline 7 & 10,10-dimethyl-2,6-dimethylidenebicyclo [7.2.0] undecan-5-ol & $\mathrm{C}_{15} \mathrm{H}_{24} \mathrm{O}$ & 0.46 & Alcohol \\
\hline 8 & Cyclohexyl isothiocyanate & $\mathrm{C}_{7} \mathrm{H}_{11} \mathrm{NS}$ & 1 & Hydrocarbon \\
\hline 9 & 3-Methoxy-4-[(trimethylsilyl)oxy] benzaldehyde 0-methyl oxime (Vanillin, MO TMS) & $\mathrm{C}_{12} \mathrm{H}_{19} \mathrm{NO}_{3} \mathrm{Si}$ & 1.85 & Aldehydes \\
\hline 10 & 8-Heptadecene & $\mathrm{C}_{17} \mathrm{H}_{34}$ & 21.9 & Hydrocarbon \\
\hline 11 & 1-Bromoheptadecane & $\mathrm{C}_{17} \mathrm{H}_{35} \mathrm{Br}$ & 2.2 & Hydrocarbon \\
\hline 12 & $3,7,11,15$-Tetramethyl-2-hexadecen-1-OL & $\mathrm{C}_{20} \mathrm{H}_{40} \mathrm{O}$ & 14.3 & Alcohol \\
\hline 13 & 6,10,14-Trimethylpentadecan-2-one & $\mathrm{C}_{18} \mathrm{H}_{36} \mathrm{O}$ & 2.4 & Ketone \\
\hline 14 & 7-Hexadecenoic acid, methyl ester, & $\mathrm{C}_{17} \mathrm{H}_{32} \mathrm{O}_{2}$ & 6.5 & Ester \\
\hline 15 & Hexadecanoic acid, methyl ester & $\mathrm{C}_{17} \mathrm{H}_{34} \mathrm{O}_{2}$ & 3.6 & Ester \\
\hline 16 & Palmitic acid & $\mathrm{C}_{16} \mathrm{H}_{32} \mathrm{O}_{2}$ & 16.2 & Fatty Acid \\
\hline 17 & Oleic acid methyl ester & $\mathrm{C}_{19} \mathrm{H}_{36} \mathrm{O}_{2}$ & 5.9 & Ester \\
\hline 18 & Phytol & $\mathrm{C}_{20} \mathrm{H}_{40} \mathrm{O}$ & 9 & Terpene \\
\hline 19 & 1,2-Benzenedicarboxylic acid, diisooctyl ester (Diisooctyl phthalate) & $\mathrm{C}_{24} \mathrm{H}_{38} \mathrm{O}_{4}$ & 2.6 & Ester \\
\hline 20 & 24Z-propylidene-cholest-5-en-3beta-ol (Methylisofucosterol) & $\mathrm{C}_{30} \mathrm{H}_{50} \mathrm{O}$ & 4.7 & Steroid \\
\hline
\end{tabular}

Table 5

Phytocomponents identified in the organic extract U. lactuca by GC-MS.

\begin{tabular}{|lllll|}
\hline No. & Compound & Molecular Formula & Peak area \% & Chemical group \\
\hline $\mathbf{1}$ & Benzoic acid, hydrazide & $\mathrm{C}_{7} \mathrm{H}_{8} \mathrm{~N}_{2} \mathrm{O}$ & 2.2 & Aldehyde \\
\hline $\mathbf{2}$ & 3-Ethyl-4-Methyl-1h-Pyrrole-2,5-Dione & $\mathrm{C}_{7} \mathrm{H}_{9} \mathrm{NO}_{2}$ & 2.5 & Ketone \\
\hline $\mathbf{3}$ & Phenol, 2,4-bis(1,1-dimethylethyl)- & $\mathrm{C}_{14} \mathrm{H}_{22} \mathrm{O}$ & 4.8 & Phenol \\
\hline $\mathbf{4}$ & Dihydroactinidiolide & $\mathrm{C}_{11} \mathrm{H}_{16} \mathrm{O}_{2}$ & 7.9 & Ketone \\
\hline $\mathbf{5}$ & Tricyclo [4.3.1.1(3,8)] undecane, 1-methoxy- & 10.2 & Hydrocarbon \\
$\mathbf{6}$ & 1,6,6-Trimethyl-7-(3-oxobut-1-enyl)-3,8-dioxatricyclo [5.1.0.0(2,4)] octan-5-one & $\mathrm{C}_{13} \mathrm{H}_{16} \mathrm{O}_{4}$ & 2.7 & Ketone \\
\hline $\mathbf{7}$ & 9,10-Dimethyltricyclo [4.2.1.1(2,5)] decane-9,10-diol & $\mathrm{C}_{12} \mathrm{H}_{20} \mathrm{O}_{2}$ & 3.7 & Alcohol \\
\hline $\mathbf{8}$ & E-2-Tetradecen-1-ol & $\mathrm{C}_{14} \mathrm{H}_{28} \mathrm{O}$ & 11.3 & Alcohol \\
$\mathbf{9}$ & Diisobutyl Phthalate & $\mathrm{C}_{16} \mathrm{H}_{22} \mathrm{O}_{4}$ & 37 & Ester \\
\hline $\mathbf{1 0}$ & Phytol & $\mathrm{C}_{20} \mathrm{H}_{40} \mathrm{O}$ & 7.7 & Terpene \\
\hline $\mathbf{1 1}$ & 7,9-Di-tert-butyl-1-oxaspiro (4,5) deca-6,9-diene-2,8-dione & $\mathrm{C}_{17} \mathrm{H}_{24}$ & 4.5 & Ketone \\
$\mathbf{1 2}$ & 1,2-Benzenedicarboxylic acid, diisooctyl ester (Diisooctyl phthalate) & $\mathrm{C}_{24} \mathrm{H}_{38} \mathrm{O}_{4}$ & 4.8 \\
\hline
\end{tabular}


Phytocomponents identified in the organic extract $A$. anceps by GC-MS.

\begin{tabular}{|lllll|}
\hline No. & Compound & Molecular Formula & Peak area \% & Chemical group \\
\hline $\mathbf{1}$ & Benzoic acid, hydrazide & $\mathrm{C}_{7} \mathrm{H}_{8} \mathrm{~N}_{2} \mathrm{O}$ & 8.1 & Aldehyde \\
\hline $\mathbf{2}$ & 2(4H)-Benzofuranone, 5,6,7,7a-tetrahydro-4,4,7a-trimethyl-, (R)- & $\mathrm{C}_{11} \mathrm{H}_{16} \mathrm{O}_{2}$ & 2.8 & Ketone \\
\hline $\mathbf{3}$ & Dodecyl acrylate & $\mathrm{C}_{15} \mathrm{H}_{28} \mathrm{O}_{2}$ & 2.2 & Ketone \\
\hline $\mathbf{5}$ & Nonadecane & $\mathrm{C}_{19} \mathrm{H}_{40}$ & 5.7 & Hydrocarbon \\
\hline $\mathbf{6}$ & Carbonic acid, butyl undec-10-enyl ester & $\mathrm{C}_{14} \mathrm{H}_{28} \mathrm{O}_{2}$ & 4.6 & Fatty acid \\
\hline 7 & 6,10,14-Trimethylpentadecan-2-one (Phytone) & $\mathrm{C}_{16} \mathrm{H}_{30} \mathrm{O}_{3}$ & 2.24 & Ester \\
\hline $\mathbf{8}$ & 7,9-Di-tert-butyl-1-oxaspiro (4,5) deca-6,9-diene-2,8-dione & $\mathrm{C}_{18} \mathrm{H}_{36} \mathrm{O}$ & 1 & Ketone \\
\hline $\mathbf{9}$ & Pentadecanoic acid, 14-methyl-, methyl ester & $\mathrm{C}_{17} \mathrm{H}_{24}$ & 3.4 & Ketone \\
\hline 10 & Palmitic acid & $\mathrm{C}_{17} \mathrm{H}_{34} \mathrm{O}_{2}$ & 29.5 & Ester \\
\hline $\mathbf{1 1}$ & 1,2-Benzenedicarboxylic acid, diisooctyl ester & $\mathrm{C}_{16} \mathrm{H}_{32} \mathrm{O}_{2}$ & 7.8 & Fatty acid \\
\hline $\mathbf{1 2}$ & Cholesterol & $\mathrm{C}_{24} \mathrm{H}_{38} \mathrm{O}_{4}$ & 30.4 & Ester \\
\hline
\end{tabular}

Table 7

Phytocomponents identified in the organic extract $C$. mediterranea by GC-MS.

\begin{tabular}{|c|c|c|c|c|}
\hline NO. & Compound & Molecular Formula & $\begin{array}{l}\text { Peak area } \\
\%\end{array}$ & Chemical group \\
\hline 1 & Benzoic acid, hydrazide & $\mathrm{C}_{7} \mathrm{H}_{8} \mathrm{~N}_{2} \mathrm{O}$ & 1.5 & Aldehyde \\
\hline 2 & Octadecanoic acid, 1-[(tetradecyloxy) carbonyl] pentadecyl ester & $\mathrm{C}_{48} \mathrm{H}_{94} \mathrm{O}_{4}$ & 1.7 & Ester \\
\hline 3 & Nonanal & $\mathrm{C}_{9} \mathrm{H}_{18} \mathrm{O}$ & 2.2 & Aldehyde \\
\hline 4 & Thiosemicarbazide & $\mathrm{CH}_{5} \mathrm{~N}_{3} \mathrm{~S}$ & 1.13 & Amide \\
\hline 5 & Sulfurous acid, butyl undecyl ester & $\mathrm{C}_{15} \mathrm{H}_{32} \mathrm{O}_{3} \mathrm{~S}$ & 2.1 & Ester \\
\hline 6 & 2,6-Difluorobenzoic acid, tridec-2-ynyl ester & $\mathrm{C}_{20} \mathrm{H}_{26} \mathrm{~F}_{2} \mathrm{O}_{2}$ & 5 & Ester \\
\hline 7 & Phenol, 3,5-bis(1,1-dimethylethyl)- & $\mathrm{C}_{14} \mathrm{H}_{22} \mathrm{O}$ & 1.76 & Phenol \\
\hline 8 & 4,4,7a-Trimethyl-5,6,7,7a-tetrahydrobenzofuran-2(4H)-one (Dihydroactinolide) & $\mathrm{C}_{11} \mathrm{H}_{16} \mathrm{O}_{2}$ & 2.26 & Ketone \\
\hline 9 & Tetradecanoic acid & $\mathrm{C}_{14} \mathrm{H}_{28} \mathrm{O}_{2}$ & 9.1 & Fatty acid \\
\hline 10 & Ethanol, 2-(9-octadecenyloxy)-, (Z)- & $\mathrm{C}_{20} \mathrm{H}_{40} \mathrm{O}_{2}$ & 3.1 & Alcohol \\
\hline 11 & Phytol & $\mathrm{C}_{20} \mathrm{H}_{40} \mathrm{O}$ & 3 & Terpene \\
\hline 12 & Diisobutyl Phthalate & $\mathrm{C}_{16} \mathrm{H}_{22} \mathrm{O}_{4}$ & 6.1 & Ester \\
\hline 13 & 7,9-Di-tert-butyl-1-oxaspiro $(4,5)$ deca-6,9-diene-2,8-dione & $\mathrm{C}_{17} \mathrm{H}_{24}$ & 8.1 & Ketone \\
\hline 14 & Pentadecanoic acid, 14-methyl-, methyl ester & $\mathrm{C}_{17} \mathrm{H}_{34} \mathrm{O}_{2}$ & 1.7 & Ester \\
\hline 15 & Ethaneperoxoic acid, 1-cyano-1-[2-(2-phenyl-1,3-dioxolan-2-yl) ethyl] pentyl ester & $\mathrm{C}_{19} \mathrm{H}_{25} \mathrm{NO}_{5}$ & 3.1 & Ester \\
\hline 16 & Hexadecane, 1,1-bis (dodecyloxy)- & $\mathrm{C}_{40} \mathrm{H}_{82} \mathrm{O}_{2}$ & 3 & Hydrocarbon \\
\hline 17 & 1-Nonadecanol & $\mathrm{C}_{19} \mathrm{H}_{40} \mathrm{O}$ & 3.7 & Alcohol \\
\hline 18 & Methyl (5Z,8Z,11Z,14Z)-icosa-5,8,11,14-tetraenoate (Methyl arachidonate) & $\mathrm{C}_{21} \mathrm{H}_{34} \mathrm{O}_{2}$ & 2.6 & Ester \\
\hline 19 & 1,2-Benzenedicarboxylic acid, diisooctyl ester & $\mathrm{C}_{24} \mathrm{H}_{38} \mathrm{O}_{4}$ & 38.2 & Ester \\
\hline
\end{tabular}




\begin{tabular}{|c|c|c|c|c|}
\hline NO. & Compound Molecular Formula & & $\begin{array}{l}\text { Peak area } \\
\%\end{array}$ & Chemical group \\
\hline 1 & Acetic acid, 2-(2-acetoxy-2,5,5,8a-tetramethyldecalin-1-yl)- & $\mathrm{C}_{18} \mathrm{H}_{30} \mathrm{O}_{4}$ & 3.8 & Aldehyde \\
\hline 2 & Oleic acid & $\mathrm{C}_{18} \mathrm{H}_{34} \mathrm{O}_{2}$ & 1.07 & Fatty acid \\
\hline 3 & Ppropiolic acid, 3-(1-hydroxy-2-isopropyl-5-methylcyclohexyl)- & $\mathrm{C}_{13} \mathrm{H}_{20} \mathrm{O}_{3}$ & 0.46 & Fatty acid \\
\hline 4 & Dihydroxanthin & $\mathrm{C}_{17} \mathrm{H}_{24} \mathrm{O}_{5}$ & 0.3 & Ketone \\
\hline 5 & Ethanol, 2-(9-octadecenyloxy)-, (Z)- & $\mathrm{C}_{20} \mathrm{H}_{40} \mathrm{O}_{2}$ & 3.07 & Alcohol \\
\hline 6 & Cholestan-3-ol, 2-methylene-, $(3 \beta, 5 a)$ - & $\mathrm{C}_{28} \mathrm{H}_{48} \mathrm{O}$ & 4 & Terpene \\
\hline 7 & 13-Heptadecyn-1-ol & $\mathrm{C}_{17} \mathrm{H}_{32} \mathrm{O}$ & 5.5 & Alcohol \\
\hline 8 & 1-Heptatriacotanol & $\mathrm{C}_{37} \mathrm{H}_{76} \mathrm{O}$ & 1.38 & Alcohol \\
\hline 9 & $\begin{array}{l}\text { Cyclopropanebutanoic acid, 2-[[2-[[2-[(2-pentylcyclopropyl) methyl] cyclopropyl] methyl] } \\
\text { cyclopropyl] methyl]-, methyl ester }\end{array}$ & $\mathrm{C}_{25} \mathrm{H}_{42} \mathrm{O}_{2}$ & 3.8 & Ester \\
\hline 10 & Palmitic acid & $\mathrm{C}_{16} \mathrm{H}_{32} \mathrm{O}_{2}$ & 0.76 & Fatty acid \\
\hline 11 & 9,12,15-Octadecatrienoic acid, 2,3-dihydroxypropyl ester, (Z, Z, Z)- & $\mathrm{C}_{21} \mathrm{H}_{36} \mathrm{O}_{4}$ & 0.46 & Ester \\
\hline 12 & Octadecanal, 2-bromo- & $\mathrm{C}_{18} \mathrm{H}_{35} \mathrm{BrO}$ & 32.5 & Hydrocarbon \\
\hline 13 & 9-Hexadecenoic acid & $\mathrm{C}_{16} \mathrm{H}_{30} \mathrm{O}_{2}$ & 0.92 & Fatty acid \\
\hline 14 & Docosahexaenoic acid, 1,2,3-propanetriyl ester & $\mathrm{C}_{69} \mathrm{H}_{98} \mathrm{O}_{6}$ & 0.61 & Ester \\
\hline 15 & Dasycarpidan-1-methanol, acetate (ester) & $\mathrm{C}_{20} \mathrm{H}_{26} \mathrm{~N}_{2} \mathrm{O}_{2}$ & 12.2 & Ester \\
\hline 16 & Retinoic acid, methyl ester & $\mathrm{C}_{21} \mathrm{H}_{30} \mathrm{O}_{2}$ & 3.3 & Ester \\
\hline 17 & $\begin{array}{l}\text { 5H-Cyclopropa [3, 4] benz[1,2-e] azulen-5-one, 9-(acetyloxy)-3- } \\
{[(\text { acetyloxy)methyl]-1,1a,1b,4,4a,7a,7b,8,9,9a-deca }}\end{array}$ & $\mathrm{C}_{24} \mathrm{H}_{32} \mathrm{O}_{8}$ & 2.3 & Ketone \\
\hline 18 & $\begin{array}{l}\text { 2-[4-methyl-6-(2,6,6-trimethylcyclohex-1-enyl) hexa-1,3,5-trienyl] cyclohex-1-en-1- } \\
\text { carboxaldehyde }\end{array}$ & $\mathrm{C}_{23} \mathrm{H}_{32} \mathrm{O}$ & 8 & Aldehyde \\
\hline 19 & Dasycarpidan-1-methanol, acetate (ester) & $\mathrm{C}_{20} \mathrm{H}_{26} \mathrm{~N}_{2} \mathrm{O}_{2}$ & 2 & Ester \\
\hline 20 & $\begin{array}{l}\text { 5H-Cyclopropa }[3,4] \text { benz }[1,2-e] \text { azulen-5-one, 4,9,9a-tris(acetyloxy)-3- } \\
{[(\text { acetyloxy)methyl]-1,1a,1b,4,4a,7a,7b,8,9, }}\end{array}$ & $\mathrm{C}_{28} \mathrm{H}_{36} \mathrm{O}_{11}$ & 1.4 & Ketone \\
\hline 21 & Phen-1,4-diol, 2,3-dimethyl-5-trifluoromethyl- & $\mathrm{C}_{9} \mathrm{H}_{9} \mathrm{~F}_{3} \mathrm{O}_{2}$ & 5.2 & Phenol \\
\hline 22 & $\begin{array}{l}\text { 5H-Cyclopropa [3,4] benz [1,2-e] azulen-5-one, 9a-(acetyloxy)-1,1a,1b,4,4a,7a,7b,8,9,9a- } \\
\text { decahydro-4a,7b,9-trihyd }\end{array}$ & $\mathrm{C}_{22} \mathrm{H}_{30} \mathrm{O}_{7}$ & 3.3 & Ketone \\
\hline 23 & $\begin{array}{l}\text { Acetic acid, 3-hydroxy-4,4,10,13-tetramethyl-7-oxo 2,3,4,7,8,9,10,11,12,13,14,15,16,17- } \\
\text { tetradecahydro-1H-cyclo }\end{array}$ & $\mathrm{C}_{23} \mathrm{H}_{34} \mathrm{O}_{4}$ & 3.7 & Hydrocarbon \\
\hline
\end{tabular}

\section{Discussion}

\subsection{Cytotoxic activity of seaweeds extracts against MCF-7 and HCT-116 cell lines}

The activity against cancer cell lines is one of the most important applications of marine algae. Many previous studies reported cytotoxic and antitumor activities of several algae (Senousy et al., 2020; Zandi et al., 2010). The SRB toxicity test represents a rapid, simple, and inexpensive assay to evaluate the cytotoxicity of extracts from natural sources such as seaweeds. The results of this assay revealed that methanol: hexane extract of all the tested seaweeds had promising cytotoxic activity against both MCF-7 and HCT-116 cancer cell lines in a concentration-dependent manner. Selection of methanol: hexane mixture for seaweeds extraction is due to its high efficiency to extract polar and nonpolar phytochemicals from seaweeds. Moreover, methanol and hexane are less toxic, less expensive, and unable to form emulsions during the extraction process (Grosso et al., 2015).

The present results agreed with Alghazeer et al. (2018) who reported significant high cytotoxic effect of $U$. lactuca extract against human colorectal carcinoma in a dose-dependent manner at a concentration range of 50-200 $\mu \mathrm{g} \mathrm{mL}^{-1}$. Ryu et al. (2013) reported that ethanol extract of $U$. fasciata collected from Korea presented a less inhibition percentage against HCT-116 (50\% at $\left.200 \mu \mathrm{g} \mathrm{mL}^{-1}\right)$ than that reported in this study $\left(56.9 \pm 1.21 \%\right.$ at $\left.50 \mu \mathrm{g} \mathrm{mL}{ }^{-1}\right)$. Moreover, ethanol extract of a green alga, Cladaphora spp. presented a promising cytotoxic activity at the concentration $7.8 \mu \mathrm{gL}^{-1} \mathrm{and}_{400 \mu \mathrm{g} \mathrm{mL}}^{-1}$ on HT-29 colon cancer 
In the present study, the extract of red alga $A$. anceps presented high cytotoxic activity on HCT-116 cancer cell line with $\mathrm{IC}_{50}$ of $13.9 \pm 1.2 \mu \mathrm{g} \mathrm{mL}^{-1}$. Harada et al. (2002) reported that the cytotoxic activity of Amphiroa zonata against human leukemic cells was due to the presence of palmitic acid. The current results declare the significant cytotoxicity of red seaweed, Corallina mediterranea against both MCF-7 and HCT-116 cancer cell lines with $\mathrm{IC}_{50}$ of $9.98 \pm 0.5 \mu \mathrm{mL}{ }^{-1}$ and $17.6 \pm 1.3 \mu \mathrm{g} \mathrm{mL}^{-1}$, respectively. Moreover, El-Kassas and El-Sheekh (2014) reported a cytotoxic effect of gold nanoparticles with aqueous extract of Corallina officinalis, collected from Egypt against human breast cancer cells.

Several Phaeophyta species are known to contain anticancer compounds mainly fucoidan, a sulfated polysaccharide. Zandi et al. (2010) reported remarkable anticancer activity of fucoidans extracted from Sargassum polycystum, S. oligocystum, S. mcclurei, S. swartzii, and S. denticaprum collected from Vietnam against human cancer cell lines. In our in vitro assay, $S$. filipendula showed low cytotoxic activity against both MCF-7 and HCT-116 cancer cell lines with IC ${ }_{50}$ of $21.2 \pm 1.1 \mu \mathrm{g} \mathrm{mL}^{-1}$ and $38.1 \pm 1.4 \mu \mathrm{g} \mathrm{mL}^{-1}$, respectively. Mary et al. (2012) reported that ethanol extract of Sargassum sp. has anticancer activity against MCF-7 cell line with an $\mathrm{IC}_{50}$ value of $250 \mu \mathrm{g} \mathrm{mL}^{-1}$. IC $\mathrm{C}_{50}$ values of $\mathrm{n}$-hexane and ethyl acetate extracts of brown seaweed, Turbinaria decurrens collected from Indonesia were $1.512 \mathrm{\mu g} \mathrm{mL}^{-1}$, and $3.058 \mathrm{\mu g} \mathrm{mL}^{-1}$, respectively. Differences in cytotoxic effect may be due to differences in seaweed species, collection site, extraction solvent, and assay methods (Zakaria et al., 2018).

The anticancer activity of the tested seaweeds may be related to the presence of various therapeutic compounds that can induce apoptosis through different pathways and molecular mechanisms (Moghadamtousi et al., 2014). Therefore, the presence of natural compounds like carbohydrates, alcohols, lipids, alkynes, amines, sulfur compounds, and sulfates in methanol: hexane extract of U. fasciata, U. lactuca, A. anceps, C. mediterranea, and $S$. filipendula may be responsible for the cytotoxic abilities of the tested seaweeds against both MCF-7 and HCT-116 cancer cell lines.

\subsection{Identification of bioactive compounds in the seaweed extracts 4.2.1. FTIR characterization of powdered seaweeds}

Marine seaweeds are a natural source of bioactive compounds with a broad range of biological activities. Until now, chemical anticancer drugs have side effects and drug interactions, and there is no natural, safe, and cheap medicine with no side effects. Thus, there is a great need to exploit novel, safe, natural anticancer agents and we think that natural products from seaweeds are ideal candidates as anticancer agents. The present study is the first to analyze the composition of Egyptian seaweeds, Ulva fasciata, Ulva lactuca, Amphiroa anceps, Corallina mediterranea, and Sargassum filipendula using FTIR, and to examine in vitro anticancer potential of their methanol: hexane extracts against MCF-7 and HCT-116 cells.

In the present study, the FT-IR results of the crude seaweeds showed the presence of various chemical groups. The strong absorption bands at 3349-3448 $\mathrm{cm}^{-1}$ in different seaweeds representing the $\mathrm{O}-\mathrm{H}$ stretching are indicative of the presence of alcohols (Hu et al., 2016; Silva et al., 2014). The weak absorption bands at 2900-2923 $\mathrm{cm}^{-1}$ in all seaweeds were due to $\mathrm{CH}_{2}$ antisymmetric stretch of methyl groups in lipids (Lu and Rasco, 2012). Noda et al. (1990) found that several glycolipids and phospholipids from seaweeds were effective against Ehrlich carcinoma.

The medium band of $A$. anceps at $2524 \mathrm{~cm}^{-1}$ and the weak bands of $U$. fasciata and $C$. mediterranea at $2500-2523 \mathrm{~cm}^{-1}$ were referred to S-H thiols stretching in sulfur compounds. The weak absorption bands observed in Chlorophytes and Rhodophytes species at 2050-2113 $\mathrm{cm}^{-1}$ are characteristic of alkynes ( $\mathrm{C} \equiv \mathrm{C}$ stretching) (Younger, 2014). The strong band at $1642 \mathrm{~cm}^{-1}$ of the spectrum of both Chlorophytes species and weak bands around $1638-1800$ $\mathrm{cm}^{-1}$ of the spectrum of Rhodophytes and Phaeophytes species are indicative of $\mathrm{C}=\mathrm{C}$ stretching of phenyl compounds and $\mathrm{C}=\mathrm{O}$ stretching of aromatic amide I (proteins and peptides), respectively (Demir et al., 2015).

The weak band in $A$. anceps at $1508 \mathrm{~cm}^{-1}$ representing C = C stretching vibration is indicative of the lignin (Kubo and Kadla, 2005). Lopez et al. (2014) reported low content of lignin in the cell walls of seaweeds. Moreover, the protecting role of lignin against colon cancer was reported by Lu et al. (1998).

The strong absorption band in $C$. mediterranea at $1411 \mathrm{~cm}^{-1}$ is due to the presence of sulfates, whereas weak absorption bands in Ulva species and $S$. filipendula at 1421-1454 $\mathrm{cm}^{-1}$ are indicative of the presence of carboxylic acid (O-H bending) (Younger, 2014). Seaweeds were known to have high carboxylic acid content, especially, fatty acids. Deyab et al. (2012) reported in vitro anticancer activities of some fatty acids (oleic and palmitic acids) extracted from seaweeds against Ehrlich ascites carcinoma cells.

The medium band in $U$. lactuca at $1099 \mathrm{~cm}^{-1}$ reflected $\mathrm{C}-0$ stretching of carbohydrates such as starch, pectin (Singh et al., 2016). The weak band of $U$. fasciata at $1108 \mathrm{~cm}^{-1}$ is attributed to $\mathrm{ACH}_{2} \mathrm{OH}$ groups of carbohydrate (Mordechai et al., 2001). Many previous studies reported that a sulfated polysaccharide, fucoidan from brown seaweeds, Fucus sp., Ascophyllum nodosum, and Undaria pinnatifida could inhibit the growth of colon cancer cells and a wide range of tumor cells (Yang et al., 2013; Kim et al., 2010).

The medium absorption bands at 1028 and $1034 \mathrm{~cm}^{-1}$ in Rhodophytes, $A$. anceps, and $C$. mediterranea, respectively referred to $\mathrm{C}-\mathrm{N}$ stretching indicate amines in these seaweeds. A. anceps, and C. mediterranea exhibited a strong band at $876 \mathrm{~cm}^{-1}$, which is assigned to $\mathrm{C}-\mathrm{H}$ bending that are characteristic of $1,2,4-$ trisubstituted compounds. The medium absorption bands at $718 \mathrm{~cm}^{-1}$ (in Rhodophytes), and weak absorption bands at $669-714 \mathrm{~cm}^{-1}$ (Chlorophytes and Phaeophytes) represent the $\mathrm{C}=\mathrm{S}$ stretching is indicative of the presence of sulfides in the tested seaweeds (Younger, 2014).

\subsubsection{GC-MS analysis}

GC-MS analysis of the extracts of the tested seaweeds revealed the presence of some active compounds. The highest number of compounds (23) was identified in the organic extract of brown seaweed, S. filipendula, whereas the lowest number of compounds (12) was identified in extracts of both $U$. lactuca, 
and $A$. anceps. Previous studies also reported a higher content of bioactive substances in brown algae than red and green algae (Gupta and Abu-Ghannam, 2011; Balboa et al., 2013; Montero et al., 2014).

Bioactive compounds of seaweeds such as terpenes, and steroids were highly diverse compared to higher plants (Manilal et al., 2013). Terpenes were detected in all the tested seaweeds except in $A$. anceps extract while steroids were found in the extracts of $U$. fasciata, and $A$. anceps. Most of these compounds expressed important various biological activities such as anticancers, antivirals, antioxidants, and anti-inflammatories. Some of the identified compounds in the extracts of the tested seaweeds like palmitic acid, oleic acid, retinoic acid, dihydroactinidiolide, thiosemicarbazide, diisobutyl phthalate, and phytol having anticancer activities (Al-Sheddi et al., 2015; Jiang et al., 2017)

Marine seaweeds are rich in fatty acids that have significant bioactivity such as anticancer activity. Pacheco et al. (2018) reported the cytotoxicity effect of fatty acids extracted from some seaweeds against MCF-7 and MDA-MB-231 human breast cancer cells. In the present study, palmitic acid was detected in the extracts of $U$. fasciata, $A$. anceps, and $S$. filipendula. The second major compound in $U$. fasciata extract was palmitic acid. It was the most prevalent saturated fatty acid in all the red seaweeds. Zafaryab et al. (2019) reported that $I_{50}$ value of palmitic acid against MCF-7 cell line was $118.87 \pm 0.22 \mu \mathrm{g} \mathrm{mL} \mathrm{m}^{-1}$ for 48 hours. The high $\mathrm{IC}_{50}$ value of palmitic acid compared with that of the tested extracts reveals that seaweed extracts result in higher cytotoxicity than pure palmitic acid. Palmitic acid extracted from Amphiroa zonata (red seaweed) induces apoptosis in the human leukemic cell line MOLT-4 (Pacheco et al., 2018). Previous studies showed a considerable anticancer potential of palmitic acid as it induces cellular apoptosis in Chinese hamster ovary (CHO) cells, pancreatic $\beta$-cells, and breast cancer cell lines (Listenberger et al., 2001; Karaskov et al., 2006; Lin et al., 2012). The main different pathways involved in apoptosis signaling mediated by palmitic acid are ROS generation, de novo ceramide synthesis, nitric oxide generation, decreases in phosphatidylinositol-3-kinase, tolllike receptor 4/reactive oxygen species (ROS)/p53 pathway, and modification of mitochondrial structure or function (Shimabukuro et al., 1998; Listenberger et al., 2001; Zhang et al., 2017). Zafaryab et al. (2019) reported that treatment of MCF-7 breast cancer with palmitic acid enhanced the expression of apoptosisrelated proteins including caspase-3, 9, Bax and p53 whereas the expression of anti-apoptotic pro tein Bcl-2 was decreased. Furthermore, oleic acid was found in U. fasciata, and S. filipendula extracts. Oleic acid exhibited high anti-proliferation of breast cancer cells through proteasome inhibition, cell cycle G0/G1 arrest, glycolysis, and induction of apoptosis and autophagy via blocking the Akt/mTOR pathway (Moon et al., 2014; Dapeng et al., 2016; Jiang et al., 2017).

Retinoic acid was found in S. filipendula extract. Retinoic acid is an active metabolite of vitamin A that was found to reduce breast (MCF-7), liver (HepG2), lung, prostate, bladder, ovarian, oral, and skin cancers via cell cycle inhibiting protein (p27) and cell cycle regulator (Cdk5) with cyto-differentiating, antiproliferative, and apoptotic effects that are mediated by activation of the nuclear hormone retinoic acid receptors RARa, RAR $\beta$ and RARY (Garattini et al., 2012; Al-Sheddi et al., 2015).

Dihydroactinidiolide, a mixture of sterols (campesterol, stigmasterol, $\beta$-sitosterol) was identified in the extract of U. lactuca. Abdul Malek et al. (2009) reported a strong cytotoxic effect of dihydroactinidiolide against colorectal carcinoma cell line (HCT-116); with IC 50 of $5.0 \mu \mathrm{g} \mathrm{mL}{ }^{-1}$. The low IC 50 value of dihydroactinidiolide compared with that of the tested extracts reveals that pure dihydroactinidiolide results in higher cytotoxicity than the seaweed extracts.

GC-MS analysis of the methanol: hexane extract of $C$. mediterranea revealed the presence of thiosemicarbazide. It has an anticancer potential similar to that of triapine and methisazone (anticancer drugs). This activity was related to its ability to inhibit ribonucleotide reductase that is involved in the rate-limiting step of DNA synthesis (Arora et al., 2014).

Diisobutyl phthalate is a phthalate ester that has moderate cytotoxic activity against various cancer cell lines (Wang et al., 2012). Diisobutyl phthalate was detected in the extracts of $U$. lactuca and $C$. mediterranea.

The organic extracts of U. fasciata, U. lactuca, and C. mediterranea contain phytol. Sheeja et al. (2016) reported a potent anticancer activity of phytol purified from Gracilaria edulis against MCF-7 cell lines with no side effects.

This study sheds light on the anticancer activity of Ulva fasciata, Ulva lactuca, Amphiroa anceps, Corallina mediterranea, and Sargassum filipendula collected from Egyptian coasts against human breast adenocarcinoma cell line (MCF-7) and colorectal carcinoma cell line (HCT-116). The crude extract of U. lactuca and $A$. anceps showed the maximum activity against MCF-7 and HCT-116 cell lines, respectively. The tested seaweeds contain different anticancer compounds that will make the seaweeds a promising source for cheap and safe anticancer drugs in the future. Therefore, purification of active substances from these seaweeds and the mechanisms by which the seaweeds induce anti-proliferation activity needs to be considered in further studies to facilitate the future potential application of these novel natural anticancer agents.

\section{Declarations}

Funding The authors received no financial support for the research, authorship, and/or publication of this article.

\section{Compliance with ethical standards}

Conflict of interest The authors declare that they have no conflict of interest.

Ethical standards This article does not contain any studies with human or animal subjects.

Informed consent There is no patient care involved in this article.

Consent to participate Not applicable.

Loading [MathJax]/jax/output/CommonHTML/fonts/TeX/fontdata.js

Page 10/15 


\section{Consent for publication Not applicable.}

Authors Contributions JM contributed to the study design and to collect data. MD contributed to the study conception and design. AS contributed to prepare materials and collect data. FW contributed to collect and analyze data. All authors wrote, read, and approved the final manuscript.

Availability of data and materials Data sharing is not applicable to this article as no datasets were generated during the current study. The data that support the findings of this secondary study are available from primary studies which were all cited.

\section{References}

1. Abdul Malek SN, Shin SK, Wahab NA, Yaacob H (2009) Cytotoxic Components of Pereskia bleo (Kunth) DC. (Cactaceae) Leaves. Molecules 14(5): 17131724. https://doi.org/10.3390/molecules 14051713

2. Alghazeer R, Howell NK, El-Naili MB, Awayn N (2018) Anticancer and Antioxidant Activities of Some Algae from Western Libyan Coast. Natural Science 10(7): 232-246. https://doi.org/10.20944/preprints201609.0018.v1

3. Al-Sheddi ES, Al-Oqail MM, Saquib Q, Siddiqui MA, Musarrat J, Al-Khedhairy AA, Farshori NN (2015) Novel All Trans-Retinoic Acid Derivatives: Cytotoxicity, Inhibition of Cell Cycle Progression and Induction of Apoptosis in Human Cancer Cell Lines. Molecules 20(5): 8181-8197. https://doi.org/10.3390/molecules20058181

4. Arora S, Agarwal S, Singhal S (2014) Anticancer activities of thiosemicarbazides/thiosemicarbazones: a review. Int J Pharm Pharm Sci 6(9): $34-41$.

5. Badal S, Gallimore W, Huang G, Tzeng TRJ, Delgoda R (2012) Cytotoxic and potent CYP1 inhibitors from the marine algae Cymopolia barbata. Org Med Chem Lett 2(1): 21. https://doi.org/10.1186/2191-2858-2-21.

6. Balboa EM, Conde E, Moure A, Falqué E, Domínguez H (2013) In vitro antioxidant properties of crude extracts and compounds from brown algae. Food Chem 138(2-3): 1764-1785. https://doi.org/10.1016/j.foodchem.2012.11.026

7. Braune W, Guiry MD (2011) Seaweeds: A colour guide to common benthic green, brown and red algae of the world's oceans. Edited by A. R. G. Gatner, Königstein, Koeltz Scientific Books, Germany, pp 601

8. Dapeng F, Jianmin L, Sheng YA (2016) Oleic/palmitate induces apoptosis in human articular chondrocytes via upregulation of NOX4 expression and ROS production. Ann Clin Lab Sci 46(4): 353-359.

9. Demir P, Onde S, Severcan F (2015) Phylogeny of cultivated and wild wheat species using ATR-FTIR spectroscopy. Spectrochim Acta A Mol Biomol Spectrosc 135: 757-763. https://doi.org/10.1016/j.saa.2014.07.025

10. Deyab MA, Habbak LZ, Ward FM (2012) Antitumor activity of water extract and some fatty acids of Turbinaria ornata (Turner) J. Agardh. Egypt J Exp Biol 8(2): 199-204.

11. Deyab MA, Mofeed J, Abd El-Halim EH, Ward F (2020) Antiviral activity of five filamentous cyanobacteria against coxsackievirus B3 and rotavirus. Arch. Microbiol. 202(2): 213-223. https://doi.org/10.1007/s00203-019-01734-9

12. El-Kassas HY, El-Sheekh MM (2014) Cytotoxic Activity of Biosynthesized Gold Nanoparticles with an Extract of the Red Seaweed Corallina officinalis on the MCF-7 Human Breast Cancer Cell Line. Asian Pac J Cancer Prev 15(10): 4311-4317 https://doi.org/10.7314/apjcp.2014.15.10.4311

13. Fitzmaurice C, Akinyemiju TF, Al Lami FH, Alam T, Alizadeh-Navaei R, Allen C, et al. (2017) Global, Regional, and National Cancer Incidence, Mortality, Years of Life Lost, Years Lived with Disability, and Disability-Adjusted Life-years for 32 Cancer Groups, 1990 to 2015: A systematic analysis for the Global Burden of Disease Study. JAMA Oncol 3(4): 524-548. https://doi.org/10.1001/jamaoncol.2016.5688.

14. Garattini E, Paroni G, Terao M (2012) Retinoids and breast cancer: new clues to increase their activity and selectivity. Breast Cancer Res $14(5)$ : 111. https://doi.org/10.1186/bcr3245

15. Grosso C, Valentão P, Ferreres F, Andrade PB (2015) Alternative and Efficient Extraction Methods for Marine-Derived Compounds. Mar Drugs 13(5): 3182-3230. https://doi.org/10.3390/md13053182

16. Gupta S, Abu-Ghannam N (2011) Bioactive potential and possible health effects of edible brown seaweeds. Trends Food Sci Tech 22(6): $315-326$. https://doi.org/10.1016/j.tifs.2011.03.011

17. Harada H, Yamashita U, Kurihara H, Fukushi E, Kawabata J, Kamei Y (2002) Antitumor activity of palmitic acid found as a selective cytotoxic substance in a marine red alga. Anticancer Res 22(5): 2587- 2590.

18. Hu Y, Pan ZJ, Liao W, Li J, Gruget P, Kitts DD, Lu X (2016) Determination of antioxidant capacity and phenolic content of chocolate by attenuated total reflectance-Fourier transformed - infrared spectroscopy. Food Chem 202: 254-261. https://doi.org/10.1016/j.foodchem.2016.01.130

19. Ibrahim AS, Khaled HM, Mikhail NN, Baraka H, Kamel H (2014) Cancer incidence in Egypt: results of the national population-based cancer registry program. J Cancer Epidemiol 2014: 437971. http://dx.doi.org/10.1155/2014/437971

20. Ilhan E, Ureyen O, Alpdogan O, Senlikci A, Alay D, Gokcelli U, Meral UM (2017) Is molecular subtypes of breast cancer related with axıllary involvement? A retrospective study of 86 cases. Acta Med Mediterr 33(3): 393-839. https://doi.org/10.19193/0393-6384_2017_3_056

21. Isnansetyo A, Lutfia FNL, Nursid M, Trijoko, Susidarti RA (2017) Cytotoxicity of Fucoidan from Three Tropical Brown Algae Against Breast and Colon Cancer Cell Lines. Pharmacogn J 9(1): 14-20 https://doi.org/10.5530/pj.2017.1.3

22. Jha B, Reddy CRK, Thakur MC, Rao MU (2009) Seaweeds of India: The diversity and distribution of seaweeds of Gujarat Coast. Edited by M. Borowitzka, Springer, Dordrecht, Netherlands, pp 215.

23. Jiang L, Wang W, He Q, Wu Y, Lu Z, Sun J, Liu Z, Shao Y, Wang A (2017) Oleic acid induces apoptosis and autophagy in the treatment of Tongue Snuamnuc noll carninomac Sri Ron 711277 httnc//doi.org/10.1038/s41598-017-11842-5

Loading [MathJax]/jax/output/CommonHTML/fonts/TeX/fontdata.js

Page $11 / 15$ 
24. Karaskov E, Scott C, Zhang L, Teodoro T, Ravazzola M, Volchuk A (2006) Chronic palmitate but not oleate exposure induces endoplasmic reticulum stress, which may contribute to INS-1 pancreatic beta-cell apoptosis. Endocrinology 147(7): 3398-3407. https://doi.org/10.1210/en.2005-1494

25. Kim EJ, Park SY, Lee JY, Park JH (2010) Fucoidan present in brown algae induces apoptosis of human colon cancer cells. BMC Gastroenterol $10,96$. https://doi.org/10.1186/1471-230X-10-96

26. Kubo S, Kadla JF (2005) Hydrogen bonding in lignin: a Fourier Transform Infrared Model Compound Study. Biomacromolecules 6(5): $2815-2821$. https://doi.org/10.1021/bm050288q

27. Lin N, Chen H, Zhang H, Wan X, Su Q (2012) Mitochondrial reactive oxygen species (ROS) inhibition ameliorates palmitate-induced INS-1 beta cell death. Endocrine 42(1): 107-117. https://doi.org/10.1007/s12020-012-9633-z

28. Listenberger LL, Ory DS, Schaffer JE (2001) Palmitate-induced apoptosis can occur through a ceramide-independent pathway. J Biol Chem 276(18): 14890-14895. https://doi.org/10.1074/jbc.M010286200

29. Lopez M, Moral A, Aguado R, Campana ML, Tijero A (2014) Evaluation of bloom algae as raw material for papermaking. $13^{\text {th }}$ European Workshop on Lignocellulosics and Pulp, At Seville, Volume 1: 527-530. https://doi.org/10.13140/2.1.3259.3924

30. Lu FJ, Chu LH, Gau RJ (1998) Free radical-scavenging properties of lignin. Nutr Cancer 30(1): 31-38. https://doi.org/10.1080/01635589809514637

31. Lu X, Rasco BA (2012) Determination of antioxidant content and antioxidant activity in foods using infrared spectroscopy and chemometrics: a review. Crit Rev Food Sci Nutr 52(10): 853-875. https://doi.org/10.1080/10408398.2010.511322.

32. Manilal A, Selvin J, Sugathan S (2013) Immuno-modulatory efficacy of indian red algae, Asparagopsis taxiformis, in Penaeus monodon. Journal of Applied Aquaculture 25(1): 81-93. https://doi.org/10.1080/10454438.2013.763514

33. Mary JS, Vinotha P, Pradeep AM (2012) Screening for in vitro cytotoxic activity of seaweed, Sargassum sp. against Hep-2 and MCF-7 cancer cell lines. Asian Pac J Cancer Prev 13(12): 6073-6076. https://doi.org/0.7314/apjcp.2012.13.12.6073

34. Mofeed J (2019) Stimulating Gamma-Linolenic acid productivity by Arthrospira platensis (Spirulina platensis) under different culture conditions (temperatures, light regime, and $\mathrm{H}_{2} \mathrm{O}_{2}$ stress). Egypt Acad J Biol Sci 11(1): 89-99. https://doi.org/10.21608/EAJBSG.2019.85014

35. Mofeed J, Deyab MA, Abd El-Halim EH (2018) Anticancer activity of some filamentous cyanobacterial isolates against Hep-G2 and MCF-7 cancer cell lines. International Journal of Life Sciences 8(1): 10-17.

36. Mofeed J, Mosleh YY (2013) Toxic responses and antioxidative enzymes activity of Scenedesmusobliquus exposed to fenhexamid and atrazine, alone and in mixture. Ecotoxicol Environ Saf 95: 234 - 240. https://doi.org/10.1016/j.ecoenv.2013.05.023

37. Mofeed J, Sabry A, Deyab MA (2019) Evaluation of biochemical composition and bioactivity of two Egyptian Ulva sp.; a comparative study. Biosci Res 16(4): 3801-3811.

38. Moghadamtousi SZ, Karimian H, Khanabdali R, Razavi M, Firoozinia M, Zandi K, Abdul Kadir H (2014) Anticancer and Antitumor Potential of Fucoidan and Fucoxanthin, Two Main Metabolites Isolated from Brown Algae. Sci World J 2014: 1-10. http://dx.doi.org/10.1155/2014/768323

39. Montero L, Herrero M, Ibanez E, Cifuentes A (2014) Separation and characterization of phlorotannins from brown algae Cystoseira abies-marina by comprehensive two-dimensional liquid chromatography. Electrophoresis 35(11): 1644-1651. https://doi.org/10.1002/elps.201400133

40. Moon HS, Batirel S, Mantzoros CS (2014) Alpha linolenic acid and oleic acid additively down-regulate malignant potential and positively cross-regulate AMPK/S6 axis in OE19 and OE33 esophageal cancer cells. Metabolism 63(11): 1447-1454. https://doi.org/10.1016/j.metabol.2014.07.009

41. Mordechai S, Mordechai J, Ramesh J, Levi C, Huleihel M, Erukhimovitch V, Moser A, Kapelushnik J (2001) Application of FTIR microspectroscopy for the follow-up of childhood leukaemia chemotherapy. Proc SPIE Subsurface and Surface Sensing Technologies and Applications III 4491: 243-250. https://doi.org/10.1117/12.450167

42. Noda H, Amano H, Arashima K, Nisizawa K (1990) Antitumor activity of marine algae. Hydrobiol 204/205(1): 577-584. https://doi.org/10.1007/BF00040290

43. Pacheco BS, dos Santos MAZ, Schultze E, Martins RM, Lund RG, Seixas FK, Colepicolo P, Collares T, Paula FR, De Pereira CMP (2018) Cytotoxic activity of fatty acids from antarctic macroalgae on the growth of human breast cancer cells. Front Bioeng Biotechnol 6:185. https://doi.org/10.3389/fbioe.2018.00185

44. Ryu MJ, Kim AD, Kang KA, Chung HS, Kim HS, Suh IS, Chang WY, Hyun JW (2013) The green algae Ulva fasciata Delile extract induces apoptotic cell death in human colon cancer cells. In Vitro Cell Dev Biol Anim 49(1): 74-81. https://doi.org/10.1007/s11626-012-9547-3

45. Senousy HH, Abd Ellatif S, Ali S (2020) Assessment of the antioxidant and anticancer potential of different isolated strains of cyanobacteria and microalgae from soil and agriculture drain water. Environ Sci Pollut Res 27: 18463-18474. https://doi.org/10.1007/s11356-020-08332-z

46. Shanthi N, Murugesan S, Kotteswari M (2017) In vitro anticancer activity of methanol extract of Cladophora spp. against HT-29 cell line. IOSR J Pharm Biol Sci 12(3): 55-61. https://doi.org/10.9790/3008-1203075561

47. Sheeja L, Lakshmi D, Bharadwaj S, Sajidha Parveen K (2016) Anticancer activity of phytol purified from Gracilaria edulis against human breast cancer cell line (MCF-7). Int J Curr Sci 19(4): E 36-46.

48. Shimabukuro M, Zhou YT, Levi M, Unger RH (1998) Fatty acid-induced beta cell apoptosis: A link between obesity and diabetes. Proc Natl Acad Sci USA 95(5): 2498-2502. https://doi.org/10.1073/pnas.95.5.2498

49. Silva SD, Feliciano RP, Boas LV, Bronze MR (2014) Application of FTIR-ATR to Moscatel dessert wines for prediction of total phenolic and flavonoid contents and antioxidant capacity. Food Chem 150: 489-493. https://doi.org/10.1016/j.foodchem.2013.11.028

50. Singh RK, Kukrety A, Sharma OP, Baranwal S, Atray N, Ray SS (2016) Study of a novel phenolic-ester as antioxidant additive in lube, biodiesel and blended diesel. J Ind Eng Chem 37: 27-31. https://doi.org/10.1016/j.jiec.2016.03.029

Loading [MathJax]/jax/output/CommonHTML/fonts/TeX/fontdata.js

Page $12 / 15$ 
51. Skehan P, Storeng R, Scudiero D, Monks A, Mcmahon J, Vistica D, Warran JT, Bokesch H, Kenney S, Boyd MR (1990) New colorimetric cytotoxicity assay for anticancer-drug screening. J Natl Cancer Inst 82(13): 1107-1112. https://doi.org/10.1093/jnci/82.13.1107

52. Tavares-Carreón F, De la Torre-Zavala S, Arocha-Garza HF, Souza V, Galán-Wong LJ, Avilés-Amaut H (2020)/n vitro anticancer activity of methanolic extract of Granulocystopsis sp., a microalgae from an oligotrophic oasis in the Chihuahuan desert. PeerJ, 8: e8686 https://doi.org/10.7717/peerj.8686

53. Torre LA, Bray F, Siegel RL, Ferlay J, Lortet-Tieulent J, Jemal A (2015) Global cancer statistics, 2012. CA Cancer J Clin 65(2): 87-108. https://doi.org/10.3322/caac.21262

54. Wang T, YuanY, Wang J, Han C, Chen G (2012) Anticancer activities of constituents from the stem of Polyalthia rumphii. Pak J Pharm Sci 25(2): $353-356$.

55. Yang L, Wang P, Wang H, Li Q, Teng H, Liu Z, Yang W, Hou L, Zou X (2013) Fucoidan derived from Undaria pinnatifada induces apoptosis in human hepatocellular carcinoma SMMC-7721 cells via the ROS mediated mitochondrial pathway. Mar Drugs 11(6): 1961-1976. https://doi.org/10.3390/md11061961.

56. Younger $\mathbf{P}$ (2014) The Merck Index (15th edition). Emerald Group Publishing Limited, London, 28(8): 38-39 https://doi.org/10.1108/RR-08-2014-0224

57. Yousefi MK, Hashtroudi MS, Moradi AM, Ghasempour AR (2018) In vitro investigating of anticancer activity of focuxanthin from marine brown seaweed species. Global J Environ Sci Manage 4(1): 81-90. https://doi.org/10.22034/gjesm.2018.04.01.008

58. Zafaryab M, Fakhri KU, Khan MA, Hajela K, Rizvi MMA (2019) In vitro Assessment of cytotoxic and apoptotic potential of Palmitic acid for Breast cancer Treatment. Int J Life Sci Res 7(1): 166-174

59. Zakaria AD, Basah K, Bahtiar A (2018) Cytotoxic Activity of Extract and Active Fraction of Turbinaria decurrens Bory on Colon Cancer Cell Line HCT-116. Int J Morphol 36(3): 979-983· https://doi.org/10.4067/S0717-95022018000300979

60. Zandi K, Ahmadzadeh S, Tajbakhsh S, Rastian Z, Yousefi F, Farshadpour F, Sartavi K (2010) Anticancer activity of Sargassum oligocystom water extract against human cancer cell lines. Eur Rev Med Pharmacol Sci 14(8): 669-673.

61. Zhang Y, Xia G, Zhang Y, Liu J, Liu X, Li W, Lv Y, Wei S, Liu J, Quan J (2017) Palmitate induces VSMC apoptosis via toll like receptor (TLR)4/ROS/p53 pathway. Atherosclerosis 263: 74-81. https://doi.org/10.1016/j.atherosclerosis.2017.06.002

\section{Figures}

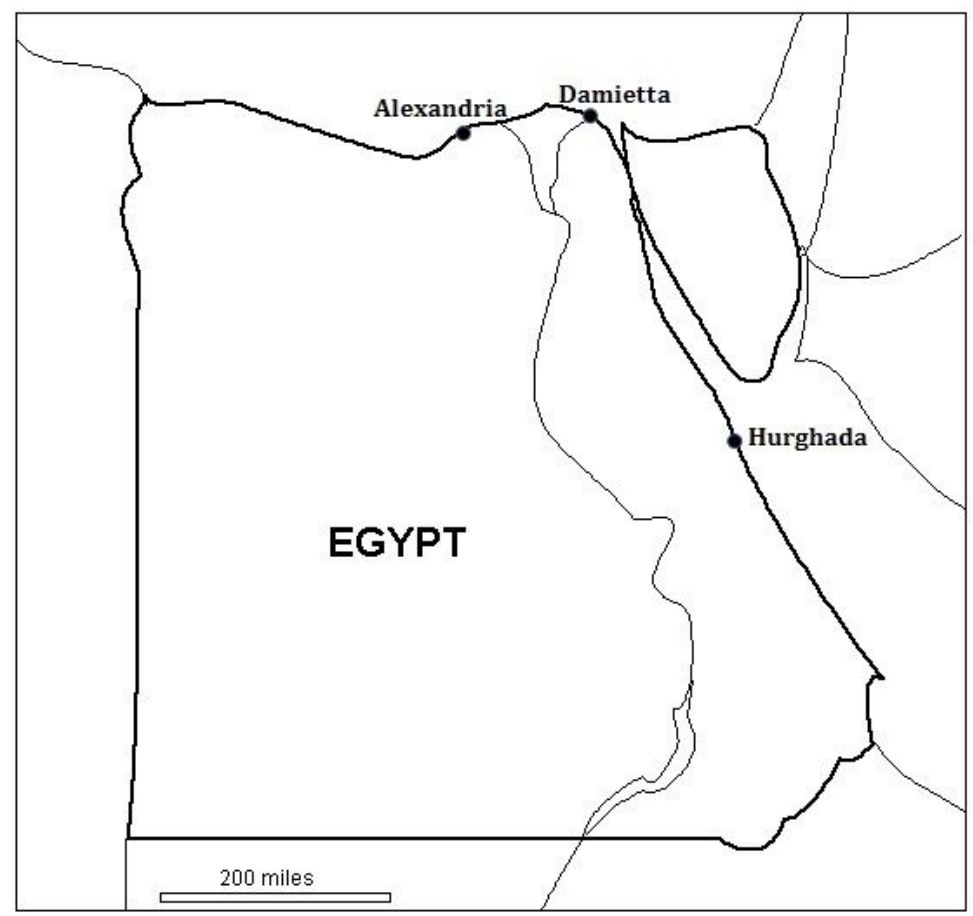

\section{Figure 1}

A map showing the collection sites. Note: The designations employed and the presentation of the material on this map do not imply the expression of any opinion whatsoever on the part of Research Square concerning the legal status of any country, territory, city or area or of its authorities, or concerning the delimitation of its frontiers or boundaries. This map has been provided by the authors. 


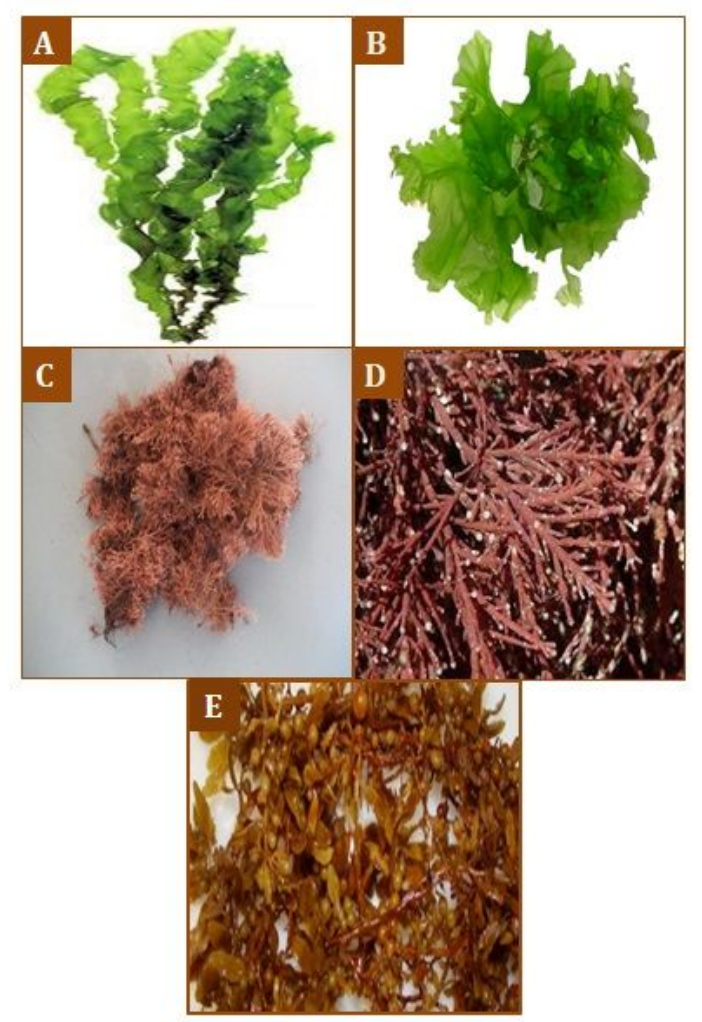

Figure 2

Photos of the collected seaweeds (A): U. fasciata, (B): U. lactuca, (C): A. anceps, (D): C. mediterranea, and (E): S. filipendula.
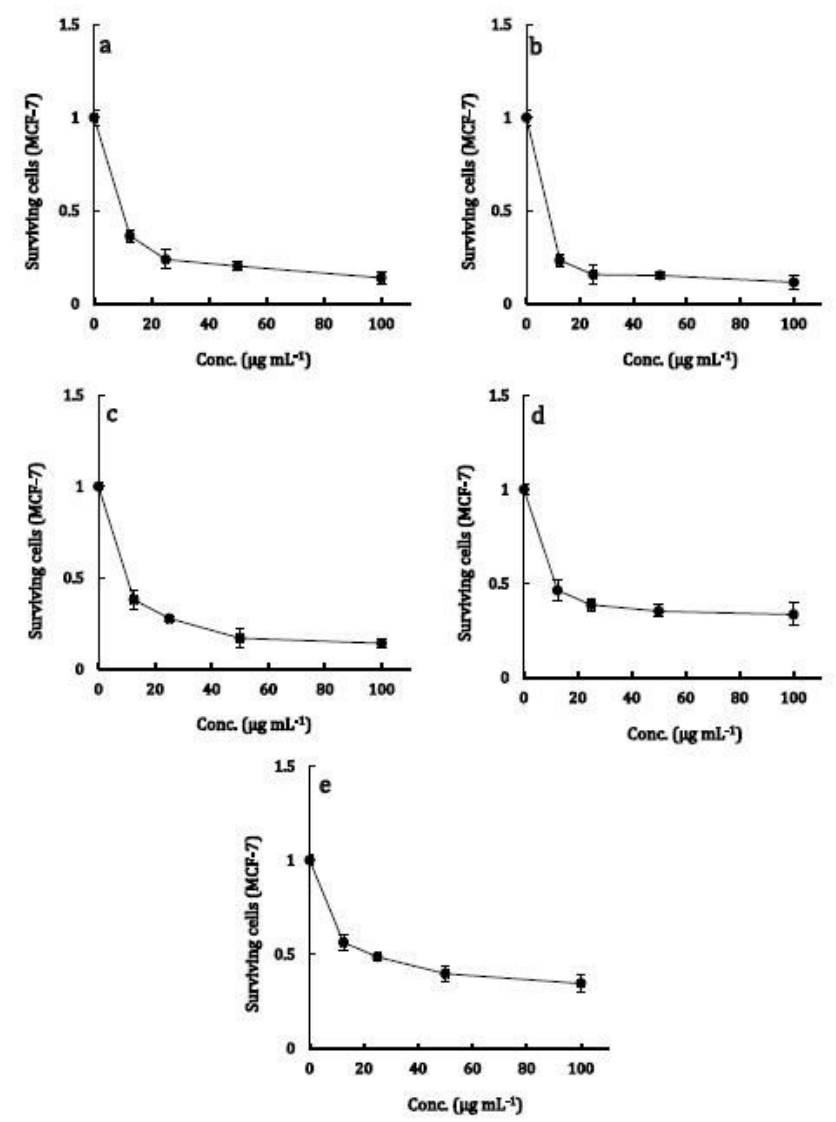

Figure 3

Effect of the organic extract of seaweeds; (a): U. fasciata, (b): U. lactuca, (c): A. anceps, (d): C. mediterranea, and (e): S. filipendula on the growth of MCF-7 Loading [MathJax]/jax/output/CommonHTML/fonts/TeX/fontdata.js tes. 

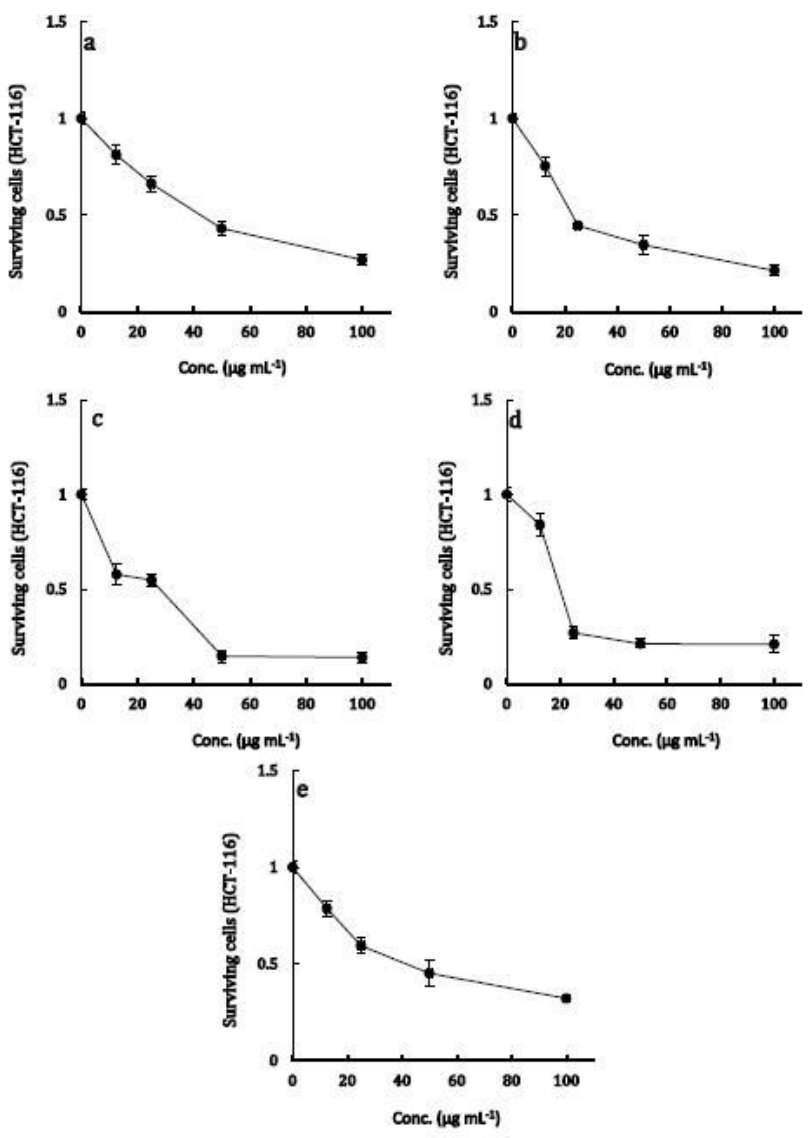

\section{Figure 4}

Effect of the organic extract of seaweeds; (a): U. fasciata, (b): U. lactuca, (c): A. anceps, (d): C. mediterranea, and (e): S. filipendula on the growth of HCT-116 cells. Results represent the means \pm SD from three replicates. 